\title{
Comparison of triple phase ct and ultrasonography findings for evaluation of hepatic lesions
}

\author{
Kaushal L ${ }^{1}$, Verma V.K ${ }^{2}$, Soni $\mathbf{N}^{3}$ \\ ${ }^{1}$ Dr. (Mrs.) Lovely Kaushal, Professor \& Head of the Department, ${ }^{2}$ Dr. V. K Verma, Associate Professor, ${ }^{3}$ Dr. Neelam \\ Soni; all authors are affiliated with Department of Radiodiagnosis and Imaging, Gandhi Medical College, Bhopal, \\ Madhya Pradesh, India.
}

Address for Correspondence: Dr. Neelam Soni, Email: sonineelam11@gmail.com

\begin{abstract}
Background: Characterizing a hepatic lesion as benign or malignant is essential for correct therapeutic plan and surgical triage. USG plays major role in screening of a liver lesion. Conventional CT with only portal venous phase has certain limitations including its inability to detect lesions which enhances in early arterial phase like HCC and those enhancing in delayed phase like Cholangiocarcinoma. Triphasic CT utilizes three phases and offers a comprehensive and accurate determination. Design: This prospective study included 100 patients with clinical suspicion of hepatic masses. Materials and Methods: All patients underwent both USG and triple phase CT, accuracy, sensitivity and specificity of both the modalities were calculated. Results: USG proved to be a good screening modality with a sensitivity of $82.7 \%$, specificity 95.6\%, PPV 82.7\% and NPV 95.6\% (p value<0.001, kappa value 0.678). Triple phase CT is excellent for characterisation and better evaluation of hepatic masses with sensitivity of $91.3 \%$, specificity $97.8 \%$, PPV $91.3 \%$ and NPV 97.8\% ( $\mathrm{p}$ value <0.001, kappa value 0.847 ). Malignant hepatic lesions can be diagnosed by triphasic CT with accuracy of $93 \%$, sensitivity and specificity of $93.3 \%$ and $92.5 \%$ respectively and with PPV and NPV of $94.9 \%$ and $90.2 \%$ respectively and by USG with accuracy of $87 \%$, sensitivity and specificity of $90 \%$ and $82.5 \%$ respectively and PPV and NPV of $88.5 \%$ and $84.6 \%$ respectively. Conclusion: Ultrasonography must be performed in all patients with clinical suspicion of hepatic masses for initial detection and localisation of lesion. Also it is widely available, less expensive and with no radiation exposure. But in comparison to triple phase CT it has lower sensitivity in differentiating benign hepatic lesions from malignant, determining accurate extension of tumor with vascular invasion.
\end{abstract}

Key words: Benign, malignant, hepatic masses, Arterial phase, Portal venous phase, Delayed phase, hepatocellular carcinoma, Cholangiocarcinoma.

\section{Introduction}

Liver is an important constituent of digestive tract. It becomes prone to various diseases because of its major function of detoxification and rich blood supply by hepatic artery and portal vein. [1,2]. Normally liver parenchyma receives about $70 \%$ of its blood from portal vein and 30\% from hepatic artery [3]. Most primary and metastatic liver tumors, receives their blood from the hepatic artery, thus reverses the normal proportion and hepatic artery becomes the prime source of blood supply. These difference in pattern of blood flow forms the basic of triple phase scan of liver [4]. This technique has helped to elucidate the imaging features of primary

Manuscript received $4^{\text {th }}$ July 2016

Reviewed: $15^{\text {th }}$ July 2016

Author Corrected: $26^{\text {th }}$ July 2016

Accepted for Publication $8^{\text {th }}$ August 2016 and metastatic liver tumors. Ultrasonography has major role in routine examination and screening of liver. It helps to determine which lesions demands further evaluation. Characterization of a hepatic lesion using USG and triple phase CT is very crucial in distinguishing a benign lesion from malignant to avoid unnecessary invasive procedures especially in benign tumors like hemangioma [5]. Improved detection and characterization can help determine which hepatic tumors may be amenable to aggressive surgical techniques and which indicate palliative treatment. Few studies have elucidated its role and most of them have been on western population. This study purports to evaluate the sonographic and triple phase CT features of common hepatic lesions with emphasis on the role of 
different phase imaging in characterization of these lesions, so that diagnosing, staging and management of patients with liver pathology could be performed more effectively.

\section{Materials and Methods}

1. Study Design: Prospective hospital based study.

2. Study Area: Gandhi Medical College and Hamidia Hospital, Bhopal. The study period was between Oct 2013 to December 2015.

3. Sample Source: Present study was conducted in the Department of Radiodiagnosis and Imaging, Bhopal. The Study population includes all the patients with suspicion of hepatic masses on clinical and/or Ultrasonography findings.

4. Sampling Methods: Simple random sampling procedure was used. Patients were selected from the attendance list of each particular day. Patients were given appointment dates depending on their convenience.

\section{Sample Size: 100 patients}

Inclusion Criteria- The study includes, Patients with suspicion of hepatic lesions on clinical and/or Ultrasonography findings.Cases of all age groups irrespective of sex.

\section{Exclusion crite ria}

- Patients with renal failure (raised serum creatinine)

- Patients who are at risk for allergic reactions to contrast.

- Pregnant patients.

- Claustrophobic patients.

- Focal liver lesions with infective etiology (hydatid cyst and liver abscess)

6. Instrumentation: All USG examinations were performed using PHILLIPS HD7 \& GE LOGIC 3 EXPERT using convex 3-5 Mhz and linear 7-12 Mhz array transducer

The computed tomographic scans were done on CT/e Wipro GE machine.

7. Method of Collection of Data: Complete evaluation of all patients was done in the following format:

- Clinical history and examination.
- Laboratory data.

- Ultrasonographic evaluation and colour doppler findings.

- Triple phase CT findings.

- Follow up/ Histopathological/post-operative findings.

All the subjects were enrolled with detailed oral and written consents. This study was approved by ethical and scientific committee of the institute.

Statistical Methods: Statistical analysis was done using computer software (SPSS IBMversion 20). Qualitative data were expressed in proportion and percentages and quantitative data expressed as mean and standard deviations. Difference in proportion was analysed by using chi square test and difference in means were analysed by using student $\mathrm{T}$ Test [unpaired]. Significance level for tests was determined as $95 \%$. Thus difference was significant if $p<0.05$.

\section{Technique of Scanning}

A. Ultrasonography: Ultrasound examination was performed in all the patients included in the study using convex and linear transducers. Acoustic gel was used for skin-transducer coupling. Patients were called in fasting state. Detailed ultrasound examination was done in multiple views like parasagittal, intercostal and subcostal oblique accordingly. Doppler examination was also done as per the requirement of the case.

B. Triple phase study: Triple phase CT scans were done in all the patients after informed consent. The exact plan of the study was individualized for each case. The patient was received in fasting state on the day of examination.

NCCT: was done initially in all the cases followed by triple phase scanning. NCCT was helpful in detecting calcification, fat and hemorrhagic areas in tumors .

CECT was obtained in three phases- Arterial, venous and equilibrium.

Arterial phase was taken at $35-40 \mathrm{sec}$ after contrast injection or 15-20 sec after bolus tracking. Lesions supplied by hepatic artery enhanced maximally in this phase. Hepatic or late portal phase was taken at 70-80 sec after contrast injection or 50-60 sec after bolustracking. Hepatic vein are enhanced in this phase with maximal enhancement of hepatic parenchyma. Hypovascular lesions were best evaluated in this phase. 
2-10 minutes after the contrast injection Delayed orequilibrium phase was obtained. Those tumors became visible in this phase that either loosed their contrast slower than normal liver parenchyma or washed out rapidly [6,7]. Contrast agent used in the study was Diatrizoatemeglumine and Diatrizoate sodium $76 \%$ both orally and iv in appropriate concentration and dosage.
Water of low density oral contrast material 1000 to $1500 \mathrm{cc}$ was given $30 \mathrm{~min}$ prior to the procedure. Dose of intravenous contrast varied according to the weight of the patient $(1.2-1.5 \mathrm{cc}$ per $\mathrm{kg})$ The injection rate was kept between $2.5-5 \mathrm{ml} / \mathrm{sec}$, rate was adjusted as such that complete contrast is administered in approximately $30 \mathrm{sec}$. (for $125 \mathrm{~mL}$ of contrast with $4 \mathrm{~mL} / \mathrm{sec}$ and for $150 \mathrm{~mL}$ with $5 \mathrm{~mL} / \mathrm{sec}$ ).

\section{Result}

In our study, population comprises of cases with age ranging from 1 year to 79 years with the maximum number of cases in the age group of 41 to 50 years (30\%). $54 \%$ of cases were males and $46 \%$ females. Largest group was formed by liver metastases with total 36 cases (36\%). Majority of cases were in age group of 41 to 50 years (44.4\%) followed by $51-60$ years $(30.5 \%)$. Second largest group was of hemangioma with total number of 23 cases (23\%) with majority of cases in the age group of $31-40$ years $(39.1 \%)$.

Table-1: Distribution of patients as per final diagnosis (based on histopathology/post-operative findings/follow-up)

\begin{tabular}{|c|c|c|c|}
\hline SN & Final Diagnosis [FD] & No of patients & \% (n=100) \\
\hline 1 & Metastasis & 36 & 36.0 \\
\hline 2 & Hemangioma & 23 & 23.0 \\
\hline 3 & HCC & 13 & 13.0 \\
\hline 4 & Cholangiocarcinoma & 9 & 9.0 \\
\hline 5 & Adenoma & 7 & 7.0 \\
\hline 6 & FNH & 3 & 3.0 \\
\hline 7 & Cirrhosis (RN) & 3.0 \\
\hline 8 & Biliary cystadenoma & 2 & 1.0 \\
\hline 9 & Hepatoblastoma & 1 & 1.0 \\
\hline 10 & Infantile hemangioendothelioma & 1 & 2.0 \\
\hline 11 & Lymphoma & 1 & 1.0 \\
\hline 12 & HepatisPeliosis & 1 & 1.0 \\
\hline
\end{tabular}

Table-2: Sex Wise Distibution.

\begin{tabular}{|c|c|c|c|}
\hline \multirow{2}{*}{ Final diagnosis } & \multicolumn{2}{|c|}{ SEX } & \multirow{2}{*}{ Total } \\
\cline { 2 - 4 } & Female & 0 & 7 \\
\hline Adenoma & 7 & 0 & 2 \\
\hline Biliary cystadenoma & 2 & 9 & 9 \\
\hline Cholangiocarcinoma & 0 & 0 & 3 \\
\hline FNH & 3 & 10 & 23 \\
\hline HCC & 3 & 7 & 13 \\
\hline Hemangioma & 16 & 1 & 1 \\
\hline Hepatic Peliosis & 0 & 1 & 1 \\
\hline Hepatoblastoma & 0 & 1 & 1 \\
\hline IHE & 0 & 1 & 36 \\
\hline Lymphoma & 0 & 21 & 3 \\
\hline Metastasis & 15 & $\mathbf{5 4}$ & \\
\hline (RN) Cirrhosis & 0 & $\mathbf{5 1 0 0}$ & \\
\hline Total & & & 2 \\
\hline
\end{tabular}


Table-3: Differentiating benign v/s malignant lesions based on USG.

\begin{tabular}{|c|c|c|c|}
\hline \multirow{2}{*}{ USG Diagnosis } & \multicolumn{2}{|c|}{ Final Diagnosis } & \multirow{2}{*}{ Total } \\
\cline { 2 - 4 } & Benign & 6 & 39 \\
\hline Benign & 33 & 54 & 61 \\
\hline Malignant & 7 & $\mathbf{6 0}$ & $\mathbf{1 0 0}$ \\
\hline Total & $\mathbf{4 0}$ & & Malignant \\
\hline
\end{tabular}

\begin{tabular}{|c|c|}
\hline p value & $<0.001$ \\
\hline Kappa & 0.729 \\
\hline Sensitivity & 82.5 \\
\hline Specificity & 90.0 \\
\hline PPV & 84.6 \\
\hline NPV & 88.5 \\
\hline Accuracy & 87.0 \\
\hline
\end{tabular}

Table-4: Differentiating benign v/s malignant lesions based on Triple phase CT.

\begin{tabular}{|c|c|c|c|}
\hline \multirow{2}{*}{ CT Diagnosis } & \multicolumn{2}{|c|}{ Final diagnosis } & \multirow{2}{*}{ Total } \\
\cline { 2 - 4 } & Benign & Malignant & 41 \\
\hline Benign & 37 & 56 & 59 \\
\hline Malignant & 3 & $\mathbf{6 0}$ & $\mathbf{1 0 0}$ \\
\hline Total & $\mathbf{4 0}$ & & 56 \\
\hline
\end{tabular}

\begin{tabular}{|c|c|}
\hline p value & $<0.001$ \\
\hline Kappa & 0.856 \\
\hline Sensitivity & 92.5 \\
\hline Specificity & 93.3 \\
\hline PPV & 90.2 \\
\hline NPV & 94.9 \\
\hline Accuracy & 87.0 \\
\hline
\end{tabular}

Table-5: Overall diagnostic values for USG and Triple phase CT

\begin{tabular}{|c|c|c|}
\hline Overall & USG & CT \\
\hline p value & $<0.001$ & 0.847 \\
\hline Kappa & 0.678 & 91.3 \\
\hline Sensitivity & 82.7 & 97.8 \\
\hline Specificity & 95.6 & 96.5 \\
\hline Accuracy & 93 & 901 \\
\hline
\end{tabular}




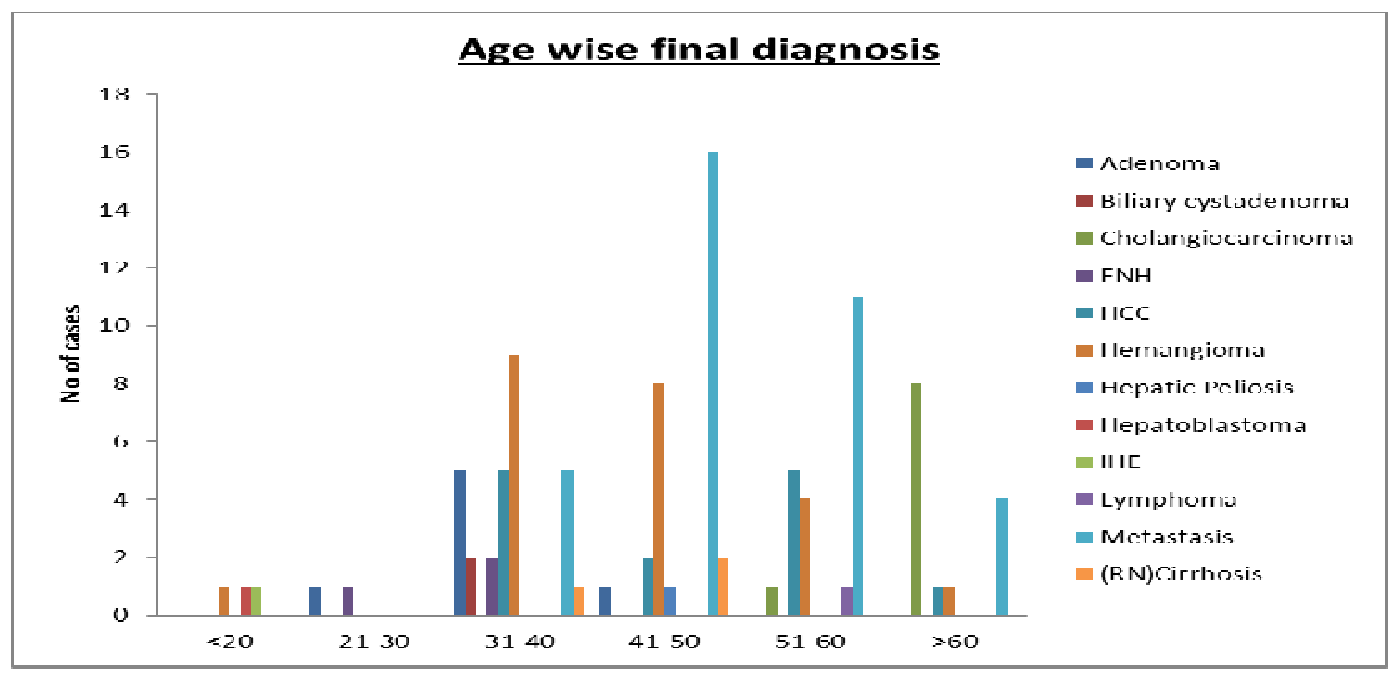

Figure-1: Age wise final diagnosis

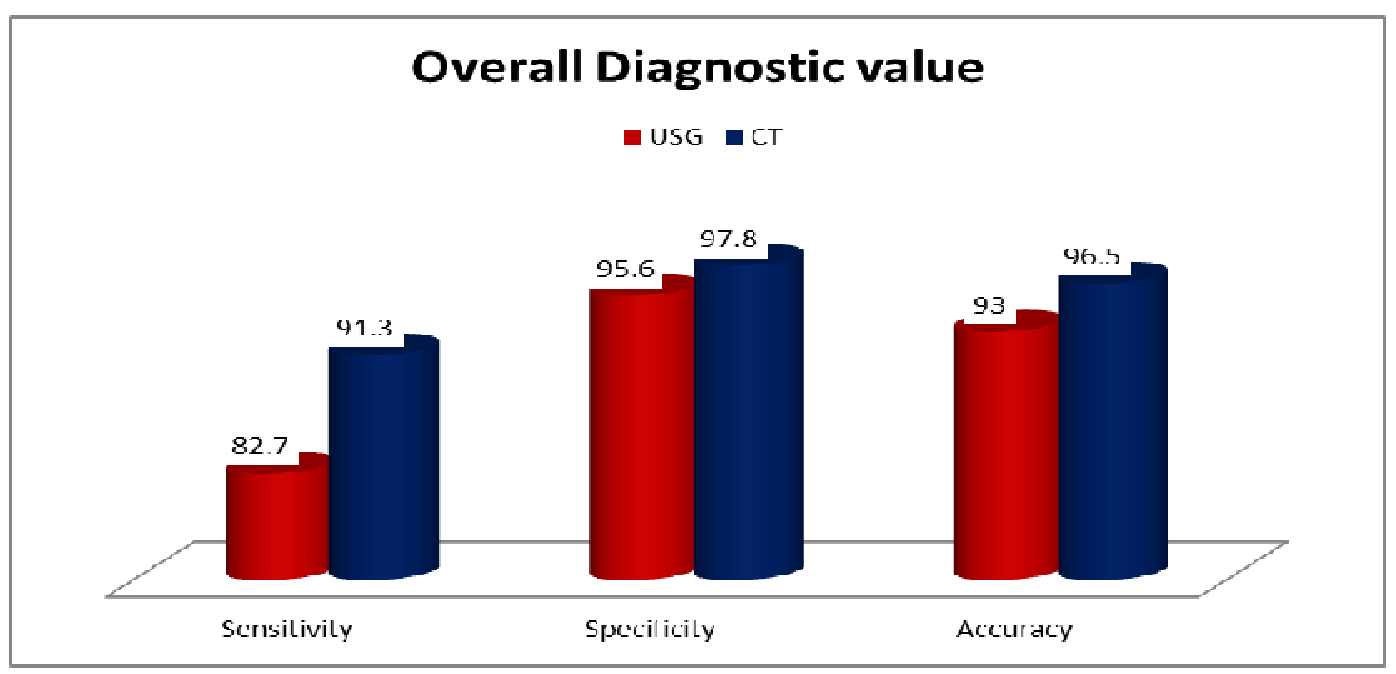

Figure-2: Overall sensitivity, specificity and accuracy of USG and CT for diagnosing a hepatic lesion.

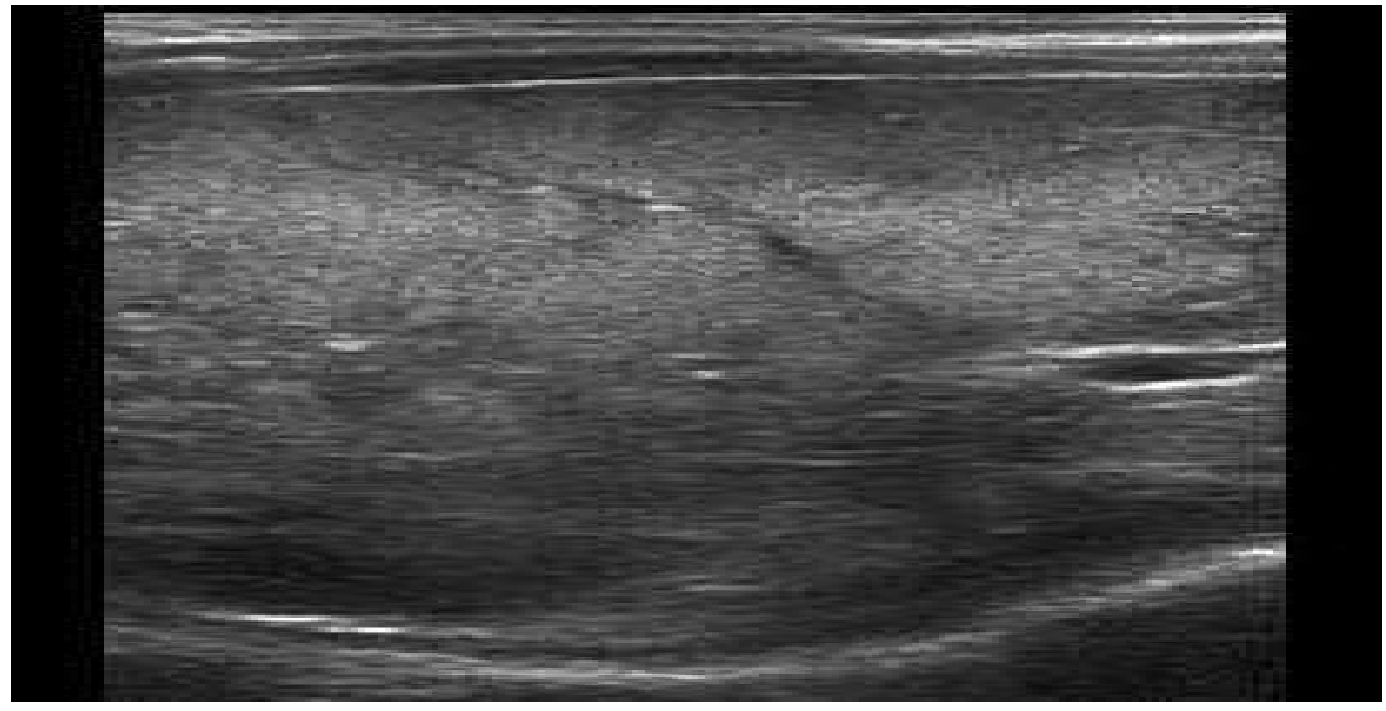

Figure-3: FNH, USG liver showing well defined hyperechoic lesion with central hypoechoic scar periphe ral enhancement of the lesion 


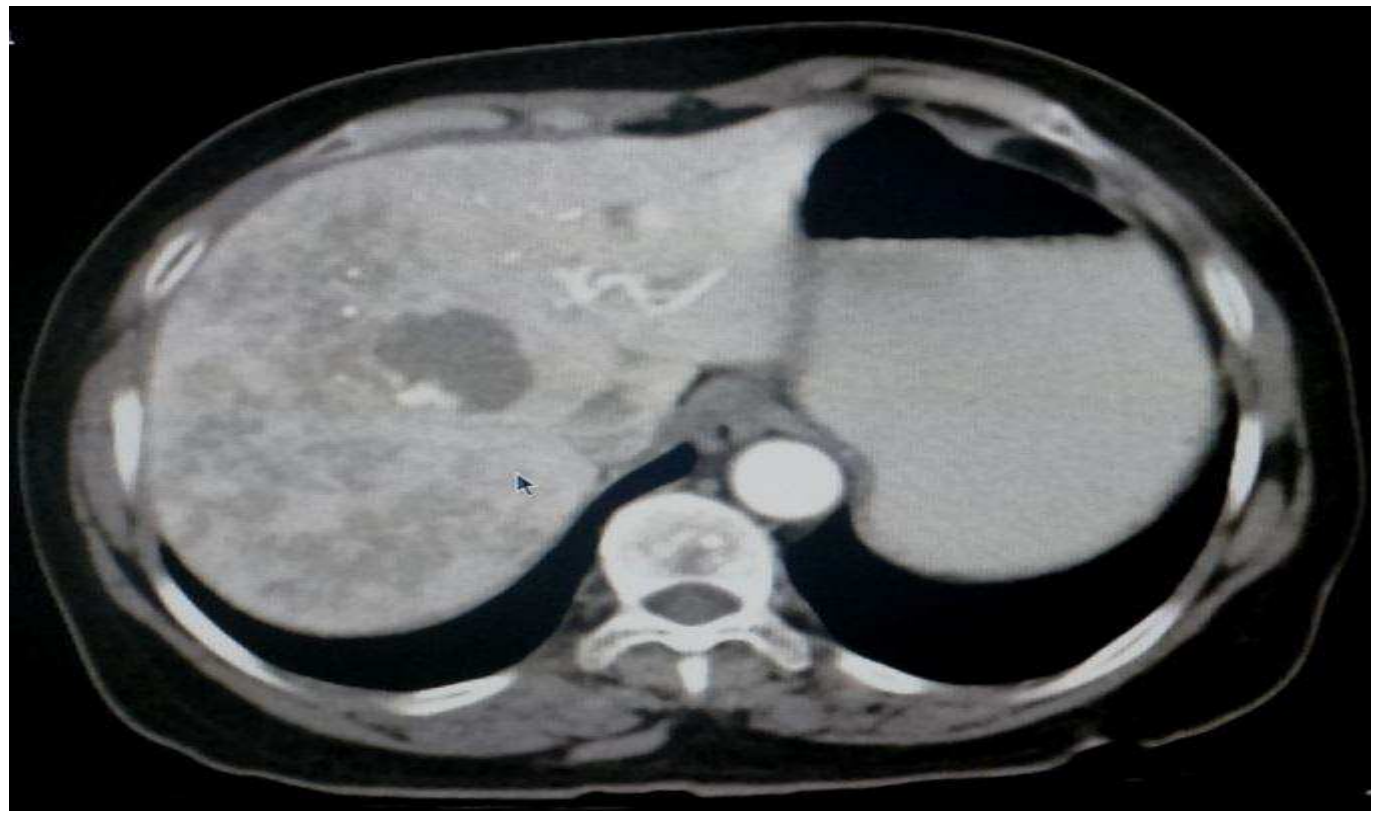

Figure-4: Hemangioma CECT abdomen, arterial phase - showing discontinuous, nodular peripheral enhancement of the lesion

Most common primary benign and malignant hepatic masses were hemangioma (23\%) and HCC (13\%) respectively. Hemangioma was commonly seen in females $(69.5 \%)$ while HCC $(76.9 \%)$ and secondary liver metastases $(58.3 \%)$ in males. Overall accuracy of diagnosing a hepatic mass by USG was found to be $93 \%$ and by triphasic CT as $96.5 \%$.

USG has been proved to be a good screening modality for diagnosing hepatic masses with a sensitivity of $82.7 \%$, specificity of $95.6 \%$, PPV of $82.7 \%$ and NPV of $95.6 \%$ (p value <0.001, kappa value 0.678 ) So ultrasonography must be performed in all patients with clinical suspicion of hepatic masses, for initial detection and localisation of lesion.

Triple phase CT is excellent for characterisation and better evaluation of hepatic masses with sensitivity of $91.3 \%$, specificity $97.8 \%$, PPV $91.3 \%$ and NPV $97.8 \%$ (p value <0.001, kappa value 0.847 ).

For better management of patients and proper surgical triage, benign lesions should be differentiated accurately and confidently from malignant. By this it can be determined that which patients demands surgery and which just needs follow up.

There were 60 malignant and 40 benign tumors in the study. Among them 56 were diagnosed correctly on triphasic CT $(94.9 \%)$.

Malignant lesions can be diagnosed by triphasic CT with an accuracy of 93\%, sensitivity and specificity of $93.3 \%$ and 92.5\% respectively and PPV and NPV of $94.9 \%$ and 90.2\% respectively. (p value < 0.001 , kappa value 0.855 ). On USG, 54 hepatic masses were correctly diagnosed as metastatic lesions $(88.5 \%)$.

Malignant lesions can be diagnosed by USG with an accuracy of $87 \%$, sensitivity and specificity of $90 \%$ and $82.5 \%$ respectively and PPV and NPV of $88.5 \%$ and $84.6 \%$ respectively. ( p value < 0.001 , kappa value 0.728 ). Metastases could be differentiated as hypervascular, hypovascular or cystic type based on triple phase CT characteristics. This further helps to define primary lesion, especially in cases of unknown primary.

33 cases of liver metastases were correctly diagnosed on triple phase CT (94.3\%). For metastases, triple phase CT has diagnostic accuracy of $95 \%$, sensitivity $91.7 \%$ and specificity $96.9 \%$ (p value $<0.001$, kappa 0.891 ). USG has diagnostic accuracy of $87 \%$, sensitivity $83.3 \%$ and specificity $89.1 \%$ (p value <0.001, kappa 0.712 ). 
22 cases were diagnosed correctly by triphasic CT (91.7\%). It has accuracy of $97 \%$, sensitivity $95.7 \%$ and specificity $97.4 \%$ for diagnosing hemangioma ( $\mathrm{p}$ value $<0.001$, kappa 0.917 ). USG has diagnostic accuracy of $93 \%$, sensitivity $82.6 \%$ and specificity $96.1 \%$ (p value $<0.001$, kappa 0.799 ).

Maximum number of cases of Adenoma were in age group 31-40 years (71.4\%). For hepatic adenoma, USG has accuracy of $96 \%$, sensitivity $71.4 \%$ and specificity $97.8 \%$ (p value $<0.001$, kappa 0.684 ) and Triphasic CT has diagnostic accuracy of $99 \%$, sensitivity $85.7 \%$ and specificity $100 \%$ (p value $<0.001$, kappa 0.799 ). Maximum cases of Cholangiocarcinoma were in age group $>60$ years $(88.8 \%) .8$ were diagnosed correctly on triphasic CT. It has diagnostic accuracy of $98 \%$, sensitivity $88.9 \%$ and specificity $98.9 \%$ (p value $<0.001$, kappa 0.823 ). USG has diagnostic accuracy of $97 \%$, sensitivity $77.8 \%$ and specificity $98.9 \%$ (p value $<0.001$, kappa 0.807 ).

For HCC Triphasic CT has diagnostic accuracy of $96 \%$, sensitivity $84.6 \%$ and specificity $97.7 \%$ (p value $<0.001$, kappa 0.823). Vascular and anatomical details of tumor were better provided by triple phase $\mathrm{CT}$ which helped to plan neoadjuvant chemotherapy and surgical or image guided intervention.

\section{Discussion}

Hemangioma- 16 cases were females and 7 males. Aytekin Oto et al 2010 have described that hemangioma are more common in females [8]. Majority of lesions $(90.9 \%)$ were of size less than $10 \mathrm{~cm}$, only 2 lesions were more than $10 \mathrm{~cm}$ and maximum number of cases had single lesion. Mayo Foundation for Medical Education and Research, 2011 has described that most of the hepatic hemangioma are small, single and do not produce symptoms. 16 lesions were hyperechoic, 5 hypoechoic, 1 had central cystic/ anechoic area and 1 was isoechoic. 20 lesions had well defined margins and 3 had ill defined margins.

17 cases were hypo dense on plain scan. On post contrast scans, all lesions showed enhancement in arterial phase (18 lesions demonstrated early discontinuous peripheral nodular enhancement while flash filling was seen in 6 cases) with progressive centripetal filling in portal venous and delayed phase. Central cystic areas were seen in 2 cases both of size more than $10 \mathrm{~cm}$. These findings were in agreement with those of Bartollota et al 2007 [9].

In 2 cases of cirrhosis regenerative nodules were misdiagnosed as hemangioma on US but on triphasic CT they were correctly diagnosed as hemangioma. Both are benign lesion and do not demand any surgical intervention. 1 case of hemangioma was misdiagnosed as adenoma on US, but on triple phase CT it revealed pathognomic features of hemangioma.

Adenoma- All the 7 cases were females and maximum in age group 31-40 years. All lesions were well defined. On USG 4 lesions were hyper echoic and 3 hypoechoic. A 36 year old female with history of hepatitis B since 4 years had well defined capsulated hypoechoic lesion in liver on USG which was diagnosed as HCC. Lesion was hypodense on plain $\mathrm{CT}$, in arterial phase lesion enhanced and became isodense in PV and delayed phase. Biopsy of the lesion was done and final diagnosis was adenoma.

Hence capsule can be present in both HCC and adenoma and should not be the differentiating criteria. According to Ichikawa $\mathrm{T}$ et al study 2000 a thin tumor capsule can be identified in approximately $25 \%$ cases [10].

FNH- All 3 cases were middle aged female. On USG all lesions were hypoechoic and demonstrated well defined margins. In 38 year old female with history of $\mathrm{Ca}$ thyroid ahypoechoic lesion in liver was diagnosed as metastatic deposit on USG. On triple phase scan, lesion enhanced in arterial phase and became isodense in PV and delayed phases. Also enhancing central scar was seen in delayed scans. Diagnosis of FNH was made on CT which was later confirmed by histopathology. Thus lesion was misdiagnosed on USG but correctly diagnosed on CT. Similar findings were described by Blachar A, Federle MP, Ferris JV study, 2002 and Stephan W. Anderson study, 2009 [11].

Another 25 year female had a small hypoechoic lesion in liver which was diagnosed as hemangiomaon USG. On triphasic CT, lesion enhanced in arterial phase which persisted in portovenous phase and became isodense in delayed phase. FNAC was done on which final diagnosis was FNH. Lesion was misdiagnosed as other benign lesion, as central scar was not well appreciated in very small sized FNH. Hence, to conclude for very small sized FNH it is not necessary that scar will be visible in $100 \%$ of the cases. 
Infantile Hemangioendothelioma- A 1 year old male had a large heterogeneous predominantly hyperechoic lesion in liver. Kassarjian A et al and Paltiel HJ et al described similar features on USG [12]. On triple phase CT it was found that the lesion was hypo dense on plain scan. On post contrast scans it showed early discontinuous peripheral enhancement on arterial phase with progressive centripetal fill-in on delayed phase. Additionally there was narrowing in calibre of infra celiac aorta [13].

Biliary Cystadenoma- Both cases were middle aged females, in age group of 31-40 years. Both had well defined hypoechoic lesion in liver. According to Levy AD Murakata LA study, 2001 biliary cystadenoma are predominantly seen in middle-aged females [14].

37 year old female with history of Ca ovary had a well defined lesion in right lobe of liver which was diagnosed as cystic metastasis. On CT, lesion demonstrated capsular enhancement in delayed phase. Final diagnosis was made as biliary cystadenoma on histopathology. According to Palacios E and Buetow PC, Midkiff RB study, on CT, they appears as a solitary cystic mass with a well-defined thick fibrous capsule. Capsular and septal enhancement is seen in post contrast scans $[15,16]$. In our study also similar findings were encountered.

Hepatocellular Carcinoma- 12 cases had lesions with well defined margins and 1 was with ill defined margins. 8 cases were hypoechoic, 5 hyperechoic and 10 with hypoechoic capsule. 1 case was misdiagnosed as metastasis in a 60 year old patient. She had a well defined hypoechoic lesion in right lobe of liver. Lesion was hypodense on plain scan and enhanced in arterial phase with early wash out in PV phase. On histopathology lesion was diagnosed as HCC.

On NCCT, 8 lesions were hypodense. All lesions showed early enhancement in arterial phase with rapid washout in portovenous phase. All lesions were hypodense in delayed phase. 10 lesions had capsular enhancement in delayed phase (76.9\%), similar findings were described by Lee et al, 2004 [17].

9 cases had portal vein thrombosis $(69.2 \%)$. Saini et al has described that the tumor thrombus is another one of the characteristic features of HCC [18]. Thus it was found that triple phase imaging with arterial, portovenous and delayed phases was advantageous in the evaluation of HCC.
Hepatoblastoma- In a two year old boy on USG a large well circumscribed lobulated mass lesion was noted in liver. The lesion was heterogeneously hyperechoic with multiple hypo and anechoic areas within it. Chung et al described similar findings [19]. Lesion was hypodense on plain CT, demonstrated heterogenous enhancement in arterial phase and become hypodense in portovenous and delayed phases [19].

Cholangiocarcinoma- All 9 cases were males and maximum ( 8 cases ) in the age group greater than 60 years. All the cases had jaundice and hyper bilirubinemia at presentation $(100 \%)$. Bloom et al has described similar features in their study [20].

All the cases in our study had single lesion, 7 lesions were hypoechoic and 2 isoechoic and all were associated with dilated IHBRD. Nisha et al have described similar USG findings [21].

3 lesions were subcapsular and all had capsular retraction. 7 lesions were hypodense and 2 isodense on NCCT and all cases demonstrated no enhancement in arterial and porto venous phase but were enhanced in delayed phase. We had similar findings as described by Nisha et al [21].

Metastases- Most of the cases (33 cases) had lesions showing well defined margins (91.6\%). 16 lesions were hypoechoic, 10 hyperechoic, target appearance was seen in 4 lesions, 4 case were cystic and 2 had calcification. 12 cases demonstrated enhancement in the arterial phase among which peripheral continuous enhancement was seen in 5 lesions and complete in 7 cases. 18 cases enhanced in portovenous phase.

All of the lesions had rapid wash out of contrast and were hypodense in delayed phase. Foley et al had described similar findings [22].

Lymphoma- A 52 year male had a well defined hypoechoic lesion in the right lobe of the liver.

Retroperitoneal and mesenteric lymphadenopathy were also noted. On USG it was diagnosed as metastatic deposit. Lesion was hypodense on plain CT and did not show enhancement in arterial and PV phase mild enhancement was observed in delayed scans after 10 minutes of contrast injection, it was diagnosed as lymphoma. Diagnosis was confirmed on histopathology as lymphoma. Thus, lesion was misdiagnosed on USG but correctly diagnosed on triphasic CT. 
According to Fazelle et al sonographically, it may either be multiple or solitary, hypo echoic or nearly anechoic mass [23]. According to Adonis Manzella et al the nodules are of low attenuation on plain CT and may show minimal enhancement [24].

\section{Conclusion}

Ultrasonography is a useful screening modality for hepatic masses with high diagnostic accuracy. Also it is cheaper, easily available and free of radiations. It is useful for initial detection and localisation of a lesion. Triple phase CT is excellent for the characterisation of hepatic masses with a diagnostic accuracy even higher than that of USG. But the patient selection for further evaluation with triple phase CT should be judicial to avoid unnecessary radiation exposure. Metastases are the most common hepatic malignancy and are far more common than primary causes like HCC. Amongst the benign lesions the most common is hemangioma. Triple phase CT is ideal for diagnosis of benign conditions like hemangioma and infantile hemangioendothelioma. Triple phase CT with its arterial, portovenous and delayed phases is an ideal modality for diagnosis and characterisation of HCC. It is helpful to provide additional information like vascular invasion, capsular delineation, arterioportal shunts and also provide a vascular road map for surgery and image guided interventions.

Pediatric malignant tumors like hepatoblastoma are diagnosed and managed with help of important information provided by triple phase CT. Vascular and tumor anatomical details are helpful to plan for neoadjuvant chemotherapy and surgical or image guided interventions. Cholangiocarcinoma is diagnosed in delayed phase images acquired during triple phase CT protocol. Metastases could be differentiated as hyper or hypovascular type based on triple phase CT characteristics. This further helps to define primary lesion and detection of unknown primary. Information derived by various phases can help in planning image guided interventions.

Recommendations -If a lesion demonstrates imaging findings diagnostic of hemangioma on both USG and triphasic CT, no further invasive procedure like biopsy is needed. If the findings are suggestive but not diagnostic of a benign lesion or if the patient is known case of primary malignancy, then follow-up imaging, further evaluation or histopathological correlation is required.
On arterial phase, many of benign as well as malignant tumors show enhancement. Thus, to correctly differentiate them and proper labelling into benign or malignant, they should be evaluated thoroughly in all the three phases and diagnosis should not be based on a single phase.

Limitations - For many rare lesions like IHE, hepatoblastoma, lymphoma sample size was small, so inadequate to calculate diagnostic accuracy of both the modalities for these lesions.

Funding: Nil, Conflict of interest: None initiated, Permission from IRB: Yes

\section{References}

1. Martini, F. H., Timmons, M. J., \&Tallitsch, R. B. (2012). Human Anatomy. (7th Edition). San Francisco: Pearson Benjamin Cummings.

2. Abdel-Misih SR, Bloomston M. Liver anatomy. Surg Clin North Am. 2010 Aug; 90 (4):643-53. doi: 10. 1016/j. suc.2010.04.017.

3. MONKHOUSE WS. Last's anatomy, regional and applied, 10 edn. Edited by C. SINNATAMBY. (Pp.X+539; f35 paperback; ISBN 044305611 0.) Edinburgh: Churchill Livingstone. 1999. Journal of Anatomy. 2000 Oct;197(3):513-8.

4. Foley WD, Mallisee TA, Hohenwalter MD, Wilson CR, Quiroz FA, Taylor AJ. Multiphase hepatic CT with a multirow detector CT scanner. AJR Am J Roentgenol. 2000 Sep;175(3):679-85.

5. Oliver JH 3rd, Baron RL, Federle MP, Rockette HE Jr. Detecting hepatocellular carcinoma: value of unenhanced or arterial phase CT imaging or both used in conjunction with conventional portal venous phase contrast-enhanced CT imaging. AJR Am J Roentgenol. 1996 Jul;167(1):71-7.

6. Atasoy C, Akyar S. Multidetector CT: contributions in liver imaging. Eur J Radiol. 2004 Oct;52(1):2-17.

7. CT and MRI of the whole body, 5th ed, 2-Vol. Set. American Journal of Neuroradiology. 2009 Apr 22; 30(7):e103-4.

8. Oto A, Kulkarni K, Nishikawa R, Baron RL. Contrast enhancement of hepatic hemangiomas on multiphase MDCT: Can we diagnose hepatic hemangiomas by 
comparing enhancement with blood pool? AJR Am J Roentgenol. 2010 Aug;195(2):381-6. doi: 10.2214/AJR. 09.3324 .

9. Bartolotta TV, Taibbi A, Galia M, Runza G, Matranga D, Midiri M, Lagalla R. Characterization of hypoechoic focal hepatic lesions in patients with fatty liver: Diagnostic performance and confidence of contrast-enhanced ultrasound. European Radiology. 2006 Oct 3;17(3):650-61.

10. Ichikawa T, Federle MP, Grazioli L, Nalesnik M. Hepatocellular adenoma: multiphasic CT and histopathologic findings in 25 patients. Radiology. 2000 Mar; 214(3):861-8.

11. Blachar A, Federle MP, Ferris JV, Lacomis JM, Waltz JS, Armfield DR, Chu G, Almusa O, Grazioli L, Balzano E, Li W. Radiologists' performance in the diagnosis of liver tumors with central scars by using specific CT criteria1. Radiology. 2002 May;223 (2):532-9.

12. Kassarjian A, Zurakowski D, Dubois J, Paltiel HJ, Fishman SJ, Burrows PE. Infantile Hepatic Hemangiomas: Clinical and imaging findings and their correlation with therapy. American Journal of Roentgenology. 2004 Mar;182(3):785-95.

13. Keslar PJ, Buck JL, Selby DM. From the archives of the AFIP. Infantile hemangioendothelioma of the liver revisited. Radiographics. 1993 May;13(3):657-70.

14. Levy AD, Murakata LA, Abbott RM, Rohrmann CA Jr. From the archives of the AFIP. Benign tumors and tumorlike lesions of the gallbladder and extrahepatic bile ducts: radiologic- pathologic correlation. Armed Forces Institute of Pathology. Radiographics. 2002 Mar-Apr;22(2):387-413.

15. Palacios E, Shannon M, Solomon C, Guzman M. Biliary cystadenoma: ultrasound, CT, and MRI. Gastrointest Radiol. 1990 Fall;15(4):313-6.
16. Mergo PJ, Helmberger TK, Buetow PC, Helmberger RC, Ros PR. Pancreatic neoplasms: MR imaging and pathologic correlation. Radiographics. 1997 Mar-Apr; 17(2):281-301.

17. Lee KH, O'Malley ME, Haider MA, Hanbidge A. Triple-phase MDCT of hepatocellular carcinoma. AJR Am J Roentgenol. 2004 Mar;182(3):643-9.

18. Hepatobiliary and Pancreatic Radiology: Imaging and intervention. Radiology. 1999 Mar;210(3):616.

19. Chung EM, Cube R, Lewis RB, Conran RM. Pediatric liver masses: Radiologic-Pathologic correlation part 1. Benign tumors1. Radio Graphics. 2010 May; 30(3):801-26.

20. Bloom CM, Langer B, Wilson SR. Role of US in the detection, characterization, and staging of cholangiocarcinoma. Radiographics. 1999 Sep-Oct; 19 (5):1199-218.

21. Sainani NI, Catalano OA, Holalkere NS, Zhu AX, Hahn PF, Sahani DV. Cholangiocarcinoma: current and novel imaging techniques. Radiographics. 2008 SepOct; 28(5):1263-87. doi: 10.1148/rg.285075183.

22. Foley WD, Kerimoglu U. Abdominal MDCT: liver, pancreas, and biliary tract. Semin Ultrasound CT MR. 2004 Apr;25(2):122-44.

23. Gazelle GS, Lee MJ, Hahn PF, Goldberg MA, Rafaat N, Mueller PR. US, CT, and MRI of primary and secondary liver lymphoma. J Comput Assist Tomogr. 1994 May-Jun;18(3):412-5.

24. Manzella A, Borba-Filho P, D'Ippolito G, Farias M. Abdominal manifestations of lymphoma: spectrum of imaging features. ISRN Radiol. 2013 Sep 2;2013:483069. doi: 10.5402/2013/483069. e Collection 2013.

\section{How to cite this article?}

Kaushal L, Verma V. K, Soni N. Comparison of triple phase ct and ultrasonography findings for evaluation of hepatic lesions. Int J Med Res Rev 2016;4(8):1456-1465.doi:10.17511/ijmrr.2016.i08.29. 